\title{
Effects of Oral Contraceptives and Metformin on The Concentrations of Anti-Müllerian Hormone in Women with Polycystic Ovary Syndrome
}

\section{Liping Wang}

Jiaxing Maternity and Child Health Care Hospital

\section{Ke Hua}

Jiaxing Maternity and Child Health Care Hospital

Wenwei Cai

Jiaxing Maternity and Child Health Care Hospital

Weiping Fu

Jiaxing Maternity and Child Health Care Hospital

Hongxia Zhang

Jiaxing Maternity and Child Health Care Hospital

Chunxia Fang ( $\nabla$ facx2015@hotmail.com )

\section{Research}

Keywords: Anti-Müllerian hormone, metformin, oral contraceptives, polycystic ovary syndrome

Posted Date: July 6th, 2020

DOl: https://doi.org/10.21203/rs.3.rs-39437/v1

License: (9) This work is licensed under a Creative Commons Attribution 4.0 International License. Read Full License 


\section{Abstract}

Background: Whether treatment with oral contraceptives (OC) and metformin can reduce anti-Müllerian hormone (AMH) levels in PCOS remains controversial. This study aims to investigate the effects of OC and metformin on serum AMH concentrations in women with polycystic ovary syndrome (PCOS).

Methods: This prospective study included 120 women with PCOS. Patients were treated with OC (35 $\mu \mathrm{g}$ of ethinyl estradiol plus $2 \mathrm{mg}$ of cyproterone acetate), metformin, or OC plus metformin for 3 months according to their different endocrine/metabolic disturbances. Forty-eight patients with hyperandrogenism $(\mathrm{HA})$ were treated with $\mathrm{OC}, 32$ patients with insulin resistance (IR) were treated with metformin, and 40 patients with HA and IR were treated with a combination of OC and metformin. Serum $\mathrm{AMH}$ levels were compared before and after treatment within each group.

Results: AMH levels decreased significantly in both OC groups (from $12.54 \pm 5.59 \mathrm{ng} / \mathrm{mL}$ before treatment to $9.03 \pm 4.49 \mathrm{ng} / \mathrm{mL}$ after treatment, $P<0.01$ ) as well as in the $\mathrm{OC}+$ metformin group (from $10.62 \pm 4.57 \mathrm{ng} / \mathrm{mL}$ to $7.74 \pm 3.19 \mathrm{ng} / \mathrm{mL}, P<0.01)$. However, AMH concentrations remained unchanged in the metformin group, although insulin sensitivity was improved.

Conclusion: Treatment with $\mathrm{OC}$ alone or $\mathrm{OC}$ plus metformin led to a significant reduction of serum AMH in PCOS patients with HA, while metformin treatment alone did not affect AMH levels in patients with IR.

\section{Plain English Summary}

This article discusses the effects of oral contraceptives(OC) and metformin on the concentrations of antiMüllerian hormone(AMH) in women with polycystic ovary syndrome(PCOS). The level of AMH is 2-3 fold higher in PCOS women compared to healthy women. The increased concentrations of AMH in PCOS is associated with $\mathrm{LH}$, total testosterone and insulin. OC suppress $\mathrm{LH}$ secretion and decrease androgen levels, whereas metformin improves insulin sensitivity. In this prospective study, women with PCOS were enrolled to be treated with OC, metformin, or OC plus metformin for 3 months according to their different endocrine /metabolic disturbances. Forty-eight patients with hyperandrogenism (HA) were treated with $\mathrm{OC}, 32$ patients with insulin resistance (IR) were treated with metformin and 40 patients with HA and IR were treated with a combination of OC and metformin. Serum AMH levels were compared before and after treatment within each group. After three months of treatment, AMH decreased significantly in both $\mathrm{OC}$ groups, as well as in the $\mathrm{OC}+$ metformin group. However, $\mathrm{AMH}$ concentrations remained unchanged in the metformin group, although insulin sensitivity was improved. Our conclusion is that treatment with OC alone or OC plus metformin led to a significant reduction of serum AMH in PCOS patients with HA, while metformin treatment did not affect AMH levels in patients with IR.

\section{Background}

PCOS is a common endocrinopathy in women and a primary cause of anovulatory infertility. It is typically characterized by HA, elevated luteinizing hormone (LH) concentrations in blood, ovulatory dysfunction, 
and polycystic ovarian morphology, but may also include insulin resistance (IR) and obesity [1]. AntiMüllerian hormone (AMH) is specifically synthetized in granulosa cells of the follicle [2]. Serum AMH is widely used as a biomarker; its use is primarily based upon its ability to assess the ovarian follicular pool [3]. The level of AMH is 2-3-fold higher in PCOS women compared with healthy women [4, 5]. According to the triangle theory by Dewailly [6], in patients with PCOS, primordial follicles grow excessively because excessive endogenous androgens make granulosa cells hypersensitive to $\mathrm{FSH}$, resulting in an increase in the number of antral follicles and overexpression of $\mathrm{AMH}$. $\mathrm{AMH}$, in turn, inhibits $\mathrm{FSH}$-induced aromatase activity, thus hindering the development of antral follicles. Although the cause of the overproduction of AMH in PCOS women remains unclear, it has been shown that androgens play a role in the excessive secretion of AMH [7]. In addition, LH significantly stimulates AMH production by granulosa cells in PCOS [4]. Many investigators have reported that the increased concentrations of AMH in PCOS is associated with $\mathrm{LH}$, total testosterone and insulin [8-10]. Therefore, AMH reflects the severity of PCOS [8, 11]. Further, it has recently been suggested that $\mathrm{AMH}$ concentration values can be used as a diagnostic and prognostic marker in PCOS patients [12, 13].

The common drugs used to treat PCOS are oral contraceptives (OC) and insulin sensitizers. OC suppress LH secretion and decrease androgen levels [14], whereas metformin, an insulin sensitizer, improves insulin sensitivity and reduces circulating androgen levels in PCOS women [15]. Several studies have evaluated the influence of these therapies on the concentrations of $\mathrm{AMH}$ in PCOS, but whether treatment with $\mathrm{OC}$ and metformin can reduce AMH levels in PCOS remains controversial. A few studies have reported a significant decrease in AMH levels during therapy with $\mathrm{OC}$ and metformin $[5,16]$; however, other studies have found no significant change in AMH levels after treatment [17-20].

In this study, we investigated the impact of $\mathrm{OC}$ and metformin on the concentration of AMH in PCOS patients.

\section{Methods}

\section{Study participants}

This study was conducted at Jiaxing Maternity and Child Health Care Hospital. In total, 141 women with PCOS (19-35 years of age) with biochemical HA and/or IR were enrolled. We adopted the 2003 Rotterdam criteria for PCOS diagnosis [21]. Total testosterone concentrations higher than the 95th percentile $\mathrm{Cl}$ of the control women $(1.97 \mathrm{nmol} / \mathrm{L})$ were considered as biochemical HA. IR was defined according to the homeostasis model assessment of IR (HOMA-IR). Patients with a HOMA-IR higher than 2.5 were considered to have IR [22]. HOMA-IR was calculated as follows: HOMA-IR = (fasting glucose $[\mathrm{mmol} / \mathrm{L}] \times$ fasting insulin [mIU/L])/22.5. Exclusion criteria included hyperprolactinemia and thyroid dysfunction, as well as other causes of HA, including Cushing syndrome, an androgen secreting tumor, and ovarian drilling or ovulation induction, prior to the study. Patients were also excluded if they wished to conceive. The ethics board of Jiaxing Maternity and Children Health Care Hospital approved this study (No. 2013-17), and all patients provided signed informed consent. 


\section{Study design}

At baseline, all patients underwent height and weight measurements, laboratory tests, and ultrasonographic examinations on the second-fourth day of the menstrual cycle. After an overnight fast, blood samples were taken from all subjects between 8:00 a.m. and 9:00 a.m. The biochemical assessments included serum AMH, LH, FSH, testosterone, estradiol (E2), insulin, and plasma glucose.

All patients received $\mathrm{OC}$, metformin, or OC plus metformin treatment for 3 months. Then, according to their different endocrine and metabolic disturbances, 141 PCOS patients were assigned to three groups, each receiving different treatments as follows.

The OC group included 56 patients with HA but no insulin resistance. Patients took an OC pill containing $35 \mu \mathrm{g}$ ethinyl E2 and $2 \mathrm{mg}$ cyproterone acetate (Diane-35®, Bayer Schering, Germany) for 21 days per cycle from the fifth day of menstruation onward.

The metformin group included 38 patients with IR but with normal levels of total testosterone. Patients were treated with $500 \mathrm{mg}$ of metformin twice a day (Glucophage, Sino-American Shanghai Squibb, China) from the first day of menstruation and with $500 \mathrm{mg}$ increments after 1 week. Progesterone was given regularly to induce uterine bleeding.

The OC + metformin group included 47 patients who exhibited not only HA but also IR. Patients were treated with a combination of OC (Diane-35) and metformin at the same doses as above.

After 3 months of therapy, clinical evaluations and hormonal and metabolic measurements were repeated on days 2-4 of the menstrual cycle. AMH levels were compared before and after treatment within each group. The flow chart of the study design is shown in Figure 1.

\section{Assays}

Plasma glucose levels were determined by an autoanalyzer (Roche Company, Germany) with the glucose oxidase method. Serum FSH, LH, E2, total testosterone, and insulin were quantified with chemiluminescence immunoassays on an Abbott Architect i2000 system (Abbott Diagnostic, Peachtree City, USA). Serum AMH concentrations were measured by an enzyme-linked immunosorbent assay (ELISA) kit (AMH ELISA; Ansh Labs, UK). The inter-assay and intra-assay coefficients of variation were $15 \%$ and $10 \%$, respectively, for $\mathrm{AMH}$.

\section{Statistical analysis}

Data were analyzed with SPSS version 18.0 software (SPSS Inc., Chicago, IL, USA). Variables are presented as mean \pm SD. A paired-sample $t$-test or a Wilcoxon signed-ranks test (in case of non-normally distributed data) was used to compare differences between baseline and post-treatment within each group. $P<0.05$ was considered statistically significant. 


\section{Results}

Of 141 PCOS patients initially enrolled in the study, 21 patients (8 patients in the OC group, 6 in the metformin group, and 7 in the $\mathrm{OC}+$ metformin group) did not complete the study (Fig. 1). Changes in endocrine and metabolic parameters in the three groups following treatment are shown in Table 1. The effects of treatments on serum AMH concentrations in the three groups are summarized in Fig. 2.

A total of 48 patients with HA received OC treatment for 3 months, reducing serum AMH levels significantly from $12.54 \pm 5.59 \mathrm{ng} / \mathrm{mL}$ to $9.03 \pm 4.49 \mathrm{ng} / \mathrm{mL}(P<0.001)$. Serum LH and testosterone concentrations also decreased significantly during treatment $(P<0.001)$. BMI and the fasting glucose and insulin levels were not significantly altered.

In the metformin group, the mean level of $A M H$ was $10.33 \pm 4.34 \mathrm{ng} / \mathrm{mL}$ at baseline and $10.13 \pm$ $4.14 \mathrm{ng} / \mathrm{mL}$ after 3 months of treatment $(P=0.144)$. Thus, AMH levels did not change significantly with metformin treatment. However, BMI, plasma glucose, serum insulin, and HOMA-IR decreased significantly $(P<0.05)$. We uncovered no significant changes in any other endocrine parameter.

With respect to the 40 patients in the $\mathrm{OC}+$ metformin group, $\mathrm{AMH}$ levels were $10.62 \pm 4.57 \mathrm{ng} / \mathrm{mL}$ at baseline, which decreased significantly to $7.74 \pm 3.19 \mathrm{ng} / \mathrm{mL}$ after treatment $(P<0.001)$. Furthermore, $\mathrm{BMI}, \mathrm{HOMA}-\mathrm{IR}$, and concentrations of $\mathrm{LH}$, testosterone, fasting glucose, and fasting insulin were also significantly decreased $(P<0.001)$.

\section{Discussion}

PCOS is a complex and heterogeneous syndrome. Women with PCOS who have HA are often treated with $\mathrm{OC}$, while those with obesity, especially with insulin resistance, may gain particular benefits from metformin therapy [11]. Treatment with $\mathrm{OC}$ and metformin can reduce androgen levels and improve insulin sensitivity; however, the influence of these treatments on serum $\mathrm{AMH}$ concentrations remains debated.

In the present study, we treated patients with $\mathrm{OC}$ and metformin according to their different endocrine and metabolic disturbances, following routine therapies used in clinical practice. It is difficult to conduct randomized controlled trials on the effects of different treatments on $\mathrm{AMH}$ in patients with PCOS, as PCOS women have heterogeneous manifestations. Thus far, there are few randomized controlled trials with large samples in this field. AMH values were compared before and after treatment within each group in this study. Importantly, self-controlled trials avoid the influence of individual differences on outcomes. Our results are reliable and have clinical implications for PCOS patients regarding these treatments. Although HA is the major feature and diagnostic component of PCOS, the clinical signs of HA might vary with ethnic differences. The rate of HA is lower in Chinese women with PCOS than in Western women. We herein selected biochemical HA as the criterion for treatment of PCOS, regardless of the symptoms of HA. 
Androgens increase the recruitment of follicles but inhibit the selection of the dominant follicle [23], and therefore women with PCOS exhibit excessive numbers of preantral and small antral follicles [24]. This excessive number of small antral follicles induced by androgens and the increase in AMH production per granulosa cell result in elevated serum AMH concentrations in PCOS patients [4, 23]. In addition, high levels of LH stimulate excessive secretion of androgen in polycystic ovaries. Bungum et al. [25] observed a significant positive co-variation between $\mathrm{LH}$ and $\mathrm{AMH}$, and this phenomenon suggested that $\mathrm{LH}$ was a possible factor involved in the control of $\mathrm{AMH}$ secretion. Consequently, $\mathrm{AMH}$ was positively correlated with testosterone and LH levels as well as total numbers of follicles [8, 9], and serum AMH levels significantly increased with HA $[8,11,26]$.

OC can inhibit LH release and reduce androgen levels. However, it remains debatable whether AMH levels decline during therapy with OC. Fábregues et al. [16] reported that AMH levels significantly decreased during $\mathrm{OC}$ treatment, explaining that the reduction in androgens and $\mathrm{LH}$ during $\mathrm{OC}$ therapy contributed to the significant decrease in serum AMH. Similar results were reported by Panidis et al. [11], who showed a significant diminution in serum AMH in women with PCOS after 6 months of treatment with OC (2 mg of cyproterone acetate plus $35 \mu \mathrm{g}$ of ethinyl E2). In our study, AMH concentrations significantly decreased after 3 months of $\mathrm{OC}$ therapy, with the reduction in $\mathrm{AMH}$ being a consequence of the suppression by OC of serum LH and testosterone levels. Our data are therefore in accordance with previous studies $[11,16]$. By contrast, Somunkiran et al. [18] did not find significant changes in AMH levels after 6 months of contraceptive treatment in women with PCOS. Our study is not consistent with that of Somunkiran et al. [18], as they did not observe a correlation between AMH levels and LH, FSH, or testosterone.

Birch et al. [27] reported that after adjusting for age, AMH was $19 \%$ lower in OC users compared with nonusers. However, the inhibitory effect of $\mathrm{OC}$ on $\mathrm{AMH}$ may be reversed within 3-6 months [28]. Based on our data and the previously aforementioned studies $[11,16]$, elevated AMH levels decreased significantly during $\mathrm{OC}$ treatment, suggesting that $\mathrm{AMH}$ is a useful indicator to evaluate $\mathrm{OC}$ treatment efficacy in clinical practice. In the current study, BMI, serum insulin levels, and HOMA-IR were not negatively affected by OC use.

Concomitant metabolic disorders constitute another prominent feature of PCOS, including obesity, IR, and metabolic syndrome [21]. IR and hyperinsulinemia are known as important pathogenic factors for PCOS, although they are not included in the definition [1]. AMH levels were found to be positively correlated with insulin and HOMA-IR in several studies $[9,10]$, suggesting that insulin plays a role in AMH synthesis and secretion [23]. Investigators have reported controversial results regarding the influence of metformin on AMH levels in PCOS patients. Two studies showed that after treatment with metformin, AMH concentrations were significantly reduced [5,29]; however, in a study by Nascimento et al. [20], patients with IR received metformin for 8 weeks, and although serum insulin concentrations and androgen levels decreased, AMH levels did not change significantly. Nascimento et al. [20] speculated that the effect of metformin on AMH levels was associated with dosage and duration of treatment. Fleming et al. [30] found that the reduction in $\mathrm{AMH}$ levels due to metformin provided a delayed response because elevated 
serum AMH levels did not change until the second 4-month period. Two other reports showed that metformin treatment significantly decreased AMH levels in hyperinsulinemic patients [31, 32].

In summary, several studies have suggested that the reduction in $\mathrm{AMH}$ levels after metformin treatment is primarily observed in highly insulin-resistant patients with PCOS [31, 32], and that the influence on peripheral serum AMH concentrations is most likely related to the dose of metformin and the duration of treatment $[20,30]$. In the present study, even though metformin treatment significantly reduced BMI, serum insulin concentrations, and HOMA-IR, AMH levels remained unaffected. Contrary to the research by Romualdi et al. [31]. and Grigoryan et al. [32], we found that metformin treatment failed to decrease circulating AMH levels in PCOS patients with IR. In the present study, although BMI, serum insulin levels, and HOMA-IR were decreased significantly after treatment, the values for these parameters were still abnormal. Serum AMH levels dropped from $10.33 \pm 4.34 \mathrm{ng} / \mathrm{mL}$ to $10.13 \pm 4.14 \mathrm{ng} / \mathrm{mL}$, suggesting a decreasing tendency after metformin treatment. Perhaps a higher dose or a longer duration of metformin treatment is needed to observe clear effects on AMH levels. Thus, the influence of metformin treatment on circulating $\mathrm{AMH}$ concentrations in PCOS women requires further study, especially with a larger sample size, in order to reach definitive conclusions.

Combined $\mathrm{OC}$ and metformin treatment is superior to $\mathrm{OC}$ alone in improving IR and is more effective in decreasing androgen levels than metformin monotherapy [33]. In clinical practice, combined treatment is more effective for those patients with obesity or IR and HA or elevated serum LH levels. However, very few data are available on the impact of the combination of $\mathrm{OC}$ and metformin therapy on AMH levels in women with PCOS. A recent study revealed that AMH levels significantly decreased in a cohort of adolescents with PCOS after treatment with metformin plus OC [34]. In our study, combined treatment resulted in a significant decrease in BMI, fasting insulin levels, and HOMA-IR. Furthermore, serum LH and testosterone levels significantly declined. Because AMH levels were positively correlated with both $\mathrm{LH}$ and testosterone levels, and $\mathrm{LH}$ and testosterone levels were significantly reduced through the use of $\mathrm{OC}$ in the combination treatment, serum AMH levels also significantly decreased. In the combination treatment, metformin did not appear to induce an additional change in AMH levels; thus, our findings were similar to those of a previous study [34]. The combined treatment was shown to improve the endocrine disorder status and metabolic aspects and to decrease serum AMH levels in PCOS. We provide additional evidence to support previous reports that AMH concentrations reflect the severity of PCOS $[8,11]$. However, the sample size of our study was small, and further studies are needed to confirm the effect of combined treatment on $\mathrm{AMH}$.

In clinical practice, treatment of women who have PCOS with clomiphene (CC) or exogenous gonadotropins for ovulation induction carries a risk of multifollicular development, resulting in ovarian hyperstimulation and multiple pregnancy $[35,36]$. Moreover, cancelation of cycles may be required due to either a poor or an excessive ovarian response. If pretreatment with $\mathrm{OC}$ and metformin decreases serum $\mathrm{AMH}$ concentrations, and if the value of $\mathrm{AMH}$ is useful for estimating treatment efficiency, then AMH may facilitate the optimal determination of subsequent ovulation induction with $\mathrm{CC}$ or gonadotropins for women with PCOS [37]. Our findings therefore expand the clinical utility of AMH. 
The limitations of our study include the small sample size and the short duration of treatment. Further studies with a larger sample size and longer treatment duration are needed to confirm the impacts of these treatments on serum $\mathrm{AMH}$ concentrations.

\section{Conclusion}

According to the endocrine / metabolic disorders in patients with PCOS, this study explored the effect of different treatment on serum $\mathrm{AMH}$ concentration. Treatment with $\mathrm{OC}$ alone or $\mathrm{OC}$ plus metformin led to a significant decrease in serum AMH levels in patients with $\mathrm{HA}$, while treatment with metformin alone did not significantly affect serum AMH levels in patients with IR. The study suggested that AMH might be a useful indicator to evaluate $\mathrm{OC}$ treatment efficacy in clinical practice.

\section{Abbreviations}

PCOS: polycystic ovary syndrome; OC: oral contraceptives; AMH: anti-Müllerian hormone; HA:

hyperandrogenism; IR: insulin resistance; LH: elevated luteinizing hormone; HOMA-IR: homeostasis model assessment of insulin resistance; E2: estradiol

\section{Declarations}

\section{Acknowledgments}

We sincerely acknowledge Department of Science and Technology of Zhejiang Province for funding this research.

\section{Authors' contributions}

LPW, KH and CXF designed the study. CXF supervised data collection and conducted data analysis. WPF, WWC and HXZ were involved in the data collection. $\mathrm{KH}$ and CXF contributed to the development of the full manuscript. All authors read and approved the final manuscript.

\section{Funding}

This research was supported by the Program for Zhejiang Leading Team of S\&T Innovation, P. R. China (No. 2011R50013-18).

\section{Availability of data and materials}

The datasets analysed during the current study are available from the corresponding author on reasonable request.

\section{Ethics approval and consent to participate}


The ethics board of Jiaxing Maternity and Child Health Care Hospital approved the study. All the procedures performed in studies involving human participants were in accordance with the ethical standards of the institutional and/or national research committee and with the 1964 Helsinki Declaration and its later amendments or comparable ethical standard.

\section{Consent for publication}

Informed consent was obtained from all individual participants included in the study.

\section{Competing interests}

The authors have no conflicts of interest to disclose.

\section{Author details}

Center of Reproductive Medicine, Jiaxing Maternity and Child Health Care Hospital, College of Medicine, Jiaxing University, Jiaxing, China.

\section{References}

1. Norman RJ, Dewailly D, Legro RS, Hickey TE: Polycystic ovary syndrome.Lancet 2007, 370:685-697.

2. Jeppesen JV, Anderson RA, Kelsey TW, Christiansen SL, Kristensen SG, Jayaprakasan K, RaineFenning N, Campbell BK, Yding Andersen C: Which follicles make the most anti-Mullerian hormone in humans? Evidence for an abrupt decline in $\mathrm{AMH}$ production at the time of follicle selection.Mol Hum Reprod 2013, 19:519-527.

3. Hansen KR, Hodnett GM, Knowlton N, Craig LB: Correlation of ovarian reserve tests with histologically determined primordial follicle number.Fertil Steril 2011, 95:170-175.

4. Pellatt L, Hanna L, Brincat M, Galea R, Brain H, Whitehead S, Mason H: Granulosa cell production of anti-Mullerian hormone is increased in polycystic ovaries.J Clin Endocrinol Metab 2007, 92:240-245.

5. Piltonen T, Morin-Papunen L, Koivunen R, Perheentupa A, Ruokonen A, Tapanainen JS: Serum antiMullerian hormone levels remain high until late reproductive age and decrease during metformin therapy in women with polycystic ovary syndrome.Hum Reprod 2005, 20:1820-1826.

6. Dewailly D, Robin G, Peigne M, Decanter C, Pigny P, Catteau-Jonard S: Interactions between androgens, $\mathrm{FSH}$, anti-Mullerian hormone and estradiol during folliculogenesis in the human normal and polycystic ovary. Hum Reprod Update 2016, 22:709-724.

7. Dumont A, Robin G, Catteau-Jonard S, Dewailly D: Role of Anti-Mullerian Hormone in pathophysiology, diagnosis and treatment of Polycystic Ovary Syndrome: a review.Reprod Biol Endocrinol 2015, 13:137.

8. Piouka A, Farmakiotis D, Katsikis I, Macut D, Gerou S, Panidis D: Anti-Mullerian hormone levels reflect severity of PCOS but are negatively influenced by obesity: relationship with increased luteinizing hormone levels.Am J Physiol Endocrinol Metab 2009, 296:E238-243. 
9. Nardo LG, Yates AP, Roberts SA, Pemberton P, Laing I: The relationships between AMH, androgens, insulin resistance and basal ovarian follicular status in non-obese subfertile women with and without polycystic ovary syndrome. Hum Reprod 2009, 24:2917-2923.

10. Skalba P, Cygal A, Madej P, Dabkowska-Huc A, Sikora J, Martirosian G, Romanik M, OlszaneckaGlinianowicz M: Is the plasma anti-Mullerian hormone (AMH) level associated with body weight and metabolic, and hormonal disturbances in women with and without polycystic ovary syndrome? Eur $J$ Obstet Gynecol Reprod Biol 2011, 158:254-259.

11. Panidis D, Georgopoulos NA, Piouka A, Katsikis I, Saltamavros AD, Decavalas G, DiamantiKandarakis E: The impact of oral contraceptives and metformin on anti-Mullerian hormone serum levels in women with polycystic ovary syndrome and biochemical hyperandrogenemia.Gynecol Endocrinol 2011, 27:587-592.

12. Wiweko B, Maidarti M, Priangga MD, Shafira N, Fernando D, Sumapraja K, Natadisastra M, Hestiantoro A: Anti-mullerian hormone as a diagnostic and prognostic tool for PCOS patients.J Assist Reprod Genet 2014, 31:1311-1316.

13. Sahmay S, Aydin Y, Oncul M, Senturk LM: Diagnosis of Polycystic Ovary Syndrome: AMH in combination with clinical symptoms.J Assist Reprod Genet 2014, 31:213-220.

14. Falsetti L, Gambera A, Tisi G: Efficacy of the combination ethinyl oestradiol and cyproterone acetate on endocrine, clinical and ultrasonographic profile in polycystic ovarian syndrome. Hum Reprod 2001, 16:36-42.

15. Tang T, Lord JM, Norman RJ, Yasmin E, Balen AH: Insulin-sensitising drugs (metformin, rosiglitazone, pioglitazone, D-chiro-inositol) for women with polycystic ovary syndrome, oligo amenorrhoea and subfertility.Cochrane Database Syst Rev 2012:CD003053.

16. Fabregues F, Castelo-Branco C, Carmona F, Guimera M, Casamitjana R, Balasch J: The effect of different hormone therapies on anti-mullerian hormone serum levels in anovulatory women of reproductive age.Gynecol Endocrinol 2011, 27:216-224.

17. Streuli I, Fraisse T, Pillet C, Ibecheole V, Bischof P, de Ziegler D: Serum antimullerian hormone levels remain stable throughout the menstrual cycle and after oral or vaginal administration of synthetic sex steroids. Fertil Steril 2008, 90:395-400.

18. Somunkiran A, Yavuz T, Yucel O, Ozdemir I: Anti-Mullerian hormone levels during hormonal contraception in women with polycystic ovary syndrome. Eur J Obstet Gynecol Reprod Biol 2007, 134:196-201.

19. Madsen HN, Lauszus FF, Trolle B, Ingerslev HJ, Torring N: Impact of metformin on anti-Mullerian hormone in women with polycystic ovary syndrome: a secondary analysis of a randomized controlled trial.Acta Obstet Gynecol Scand 2015, 94:547-551.

20. Nascimento AD, Silva Lara LA, Japur de Sa Rosa-e-Silva AC, Ferriani RA, Reis RM: Effects of metformin on serum insulin and anti-Mullerian hormone levels and on hyperandrogenism in patients with polycystic ovary syndrome.Gynecol Endocrinol 2013, 29:246-249. 
21. Rotterdam EA-SPcwg: Revised 2003 consensus on diagnostic criteria and long-term health risks related to polycystic ovary syndrome (PCOS). Hum Reprod 2004, 19:41-47.

22. Reyes-Munoz E, Ortega-Gonzalez C, Martinez-Cruz N, Arce-Sanchez L, Estrada-Gutierrez G, Moran C, Sanchez-Serrano AP, Higareda-Sanchez R, de la Jara-Diaz JF: Association of obesity and overweight with the prevalence of insulin resistance, pre-diabetes and clinical-biochemical characteristics among infertile Mexican women with polycystic ovary syndrome: a cross-sectional study.BMJ Open 2016, 6:e012107.

23. Jonard S, Dewailly D: The follicular excess in polycystic ovaries, due to intra-ovarian hyperandrogenism, may be the main culprit for the follicular arrest.Hum Reprod Update 2004, 10:107-117.

24. Franks S, Stark J, Hardy K: Follicle dynamics and anovulation in polycystic ovary syndrome.Hum Reprod Update 2008, 14:367-378.

25. Bungum L, Franssohn F, Bungum M, Humaidan P, Giwercman A: The circadian variation in AntiMullerian hormone in patients with polycystic ovary syndrome differs significantly from normally ovulating women.PLoS One 2013, 8:e68223.

26. Li Y, Ma Y, Chen X, Wang W, Li Y, Zhang Q, Yang D: Different diagnostic power of anti-Mullerian hormone in evaluating women with polycystic ovaries with and without hyperandrogenism.J Assist Reprod Genet 2012, 29:1147-1151.

27. Birch Petersen K, Hvidman HW, Forman JL, Pinborg A, Larsen EC, Macklon KT, Sylvest R, Andersen AN: Ovarian reserve assessment in users of oral contraception seeking fertility advice on their reproductive lifespan. Hum Reprod 2015, 30:2364-2375.

28. van den Berg MH, van Dulmen-den Broeder E, Overbeek A, Twisk JW, Schats R, van Leeuwen FE, Kaspers GJ, Lambalk CB: Comparison of ovarian function markers in users of hormonal contraceptives during the hormone-free interval and subsequent natural early follicular phases. Hum Reprod 2010, 25:1520-1527.

29. Neagu M, Cristescu C: Anti-Mullerian hormone-a prognostic marker for metformin therapy efficiency in the treatment of women with infertility and polycystic ovary syndrome.J Med Life 2012, 5:462-464.

30. Fleming R, Harborne L, MacLaughlin DT, Ling D, Norman J, Sattar N, Seifer DB: Metformin reduces serum mullerian-inhibiting substance levels in women with polycystic ovary syndrome after protracted treatment.Fertil Steril 2005, 83:130-136.

31. Romualdi D, De Cicco S, Tagliaferri V, Proto C, Lanzone A, Guido M: The metabolic status modulates the effect of metformin on the antimullerian hormone-androgens-insulin interplay in obese women with polycystic ovary syndrome.J Clin Endocrinol Metab 2011, 96:E821-824.

32. Grigoryan O, Absatarova J, Andreeva E, Melnichenko G, Dedov I: Effect of metformin on the level of anti-Mullerian hormone in therapy of polycystic ovary syndrome in obese women.Minerva Ginecol 2014, 66:85-89.

33. Wu J, Zhu Y, Jiang Y, Cao Y: Effects of metformin and ethinyl estradiol-cyproterone acetate on clinical, endocrine and metabolic factors in women with polycystic ovary syndrome.Gynecol 
Endocrinol 2008, 24:392-398.

34. Dursun F, Guven A, Yildiz M: Assessment of Anti-Mullerian Hormone Level in Management of Adolescents with Polycystic Ovary Syndrome.J Clin Res Pediatr Endocrinol 2016, 8:55-60.

35. Balen AH, Morley LC, Misso M, Franks S, Legro RS, Wijeyaratne CN, Stener-Victorin E, Fauser BC, Norman RJ, Teede $\mathrm{H}$ : The management of anovulatory infertility in women with polycystic ovary syndrome: an analysis of the evidence to support the development of global WHO guidance. Hum Reprod Update 2016, 22:687-708.

36. Birch Petersen K, Pedersen NG, Pedersen AT, Lauritsen MP, la Cour Freiesleben N: Mono-ovulation in women with polycystic ovary syndrome: a clinical review on ovulation induction.Reprod Biomed Online 2016, 32:563-583.

37. Mumford SL, Legro RS, Diamond MP, Coutifaris C, Steiner AZ, Schlaff WD, Alvero R, Christman GM, Casson PR, Huang $\mathrm{H}$, et al: Baseline AMH Level Associated With Ovulation Following Ovulation Induction in Women With Polycystic Ovary Syndrome.J Clin Endocrinol Metab 2016, 101:3288-3296.

\section{Table}


Table 1 AMH concentrations and endocrine and metabolic parameters in the 3 groups before and after 3 months of treatment

\begin{tabular}{|c|c|c|c|c|c|c|c|c|c|}
\hline & \multicolumn{3}{|c|}{ OC group $(n=48)$} & \multicolumn{3}{|c|}{ metformin group $(n=32)$} & \multicolumn{3}{|c|}{$\mathrm{OC}+$ metformin group $(\mathrm{n}=40)$} \\
\hline & $\begin{array}{c}\text { Pre-treat } \\
\text { ment }\end{array}$ & $\begin{array}{c}\text { Post-treat } \\
\text { ment }\end{array}$ & $P$ & $\begin{array}{c}\text { Pre-treat } \\
\text { ment }\end{array}$ & $\begin{array}{c}\text { Post-treat } \\
\text { ment }\end{array}$ & $P$ & $\begin{array}{c}\text { Pre-treatm } \\
\text { ent }\end{array}$ & $\begin{array}{c}\text { Post-treat } \\
\text { ment }\end{array}$ & $P$ \\
\hline BMI & $21.04 \pm 2$ & $21.10 \pm 2.4$ & 0.6 & $27.32 \pm 3.7$ & $25.95 \pm 3.2$ & 0.0 & $27.49 \pm 3.8$ & $26.16 \pm 3.0$ & 0.0 \\
\hline$\left(\mathrm{kg} / \mathrm{m}^{2}\right)$ & 62 & 4 & 66 & 9 & 1 & 00 & 3 & 6 & 00 \\
\hline FSH & $4.87 \pm 0.9$ & $4.82 \pm 0.98$ & 0.7 & $4.59 \pm 1.04$ & $4.70 \pm 0.94$ & 0.5 & $4.61 \pm 1.12$ & $4.49 \pm 1.08$ & 0.4 \\
\hline (IU/L) & 3 & & 10 & & & 60 & & & 03 \\
\hline \multirow[t]{2}{*}{$\mathrm{LH}(\mathrm{IU} / \mathrm{L})$} & $11.32 \pm 4.6$ & $6.25 \pm 2.80$ & 0.0 & $4.65 \pm 2.50$ & $4.17 \pm 1.52$ & 0.2 & $7.21 \pm 3.32$ & $4.32 \pm 2.04$ & 0.0 \\
\hline & 7 & & 00 & & & 93 & & & 00 \\
\hline $\mathrm{T}$ & $2.24 \pm 0.2$ & $1.60 \pm 0.42$ & 0.0 & $1.35 \pm 0.39$ & $1.30 \pm 0.36$ & 0.3 & $2.36 \pm 0.39$ & $1.50 \pm 0.50$ & 0.0 \\
\hline$(\mathrm{nmol} / \mathrm{L})$ & 8 & & 01 & & & 48 & & & 00 \\
\hline Glucose & $5.09 \pm 0.3$ & $5.08 \pm 0.36$ & 0.7 & $5.32 \pm 0.46$ & $5.19 \pm 0.38$ & 0.0 & $5.51 \pm 0.39$ & $5.22 \pm 0.38$ & 0.0 \\
\hline$(\mathrm{mmol} / \mathrm{L}$ & 3 & & 56 & & & 36 & & & 00 \\
\hline \multicolumn{10}{|l|}{ ) } \\
\hline Insulin & $53.45 \pm 17$ & $55.54 \pm 15$ & 0.9 & $149.67 \pm 5$ & $96.82 \pm 22$ & 0.0 & $151.77 \pm 10$ & $104.87 \pm 55$ & 0.0 \\
\hline$(\mathrm{pmol} / \mathrm{L})$ & .28 & 87 & 59 & 4.67 & 80 & 00 & 2.31 & .72 & 00 \\
\hline HOMA-I & $1.74 \pm 0.5$ & $1.80 \pm 0.52$ & 0.7 & $5.10 \pm 1.97$ & $3.22 \pm 0.86$ & 0.0 & $5.38 \pm 3.77$ & $3.51 \pm 1.88$ & 0.0 \\
\hline $\mathrm{R}$ & 9 & & 58 & & & 00 & & & 00 \\
\hline $\mathrm{AMH}(\mathrm{ng} /$ & $12.54 \pm 5$ & $9.03 \pm 4.49$ & 0.0 & $10.33 \pm 4.3$ & $10.13 \pm 4.1$ & 0.3 & $10.62 \pm 4.5$ & $7.74 \pm 3.19$ & 0.0 \\
\hline $\mathrm{ml})$ & 59 & & 00 & 4 & 4 & 30 & 7 & & 00 \\
\hline
\end{tabular}

OC, oral contraceptive; BMI, body mass index; FSH, follicle-stimulating hormone; LH, luteinizing hormone; T, testosterone; HOMA-IR, homeostatic model assessment of insulin sensitivity; AMH, antimullerian hormone; Values are means $\pm \mathrm{SD}$

Figures 


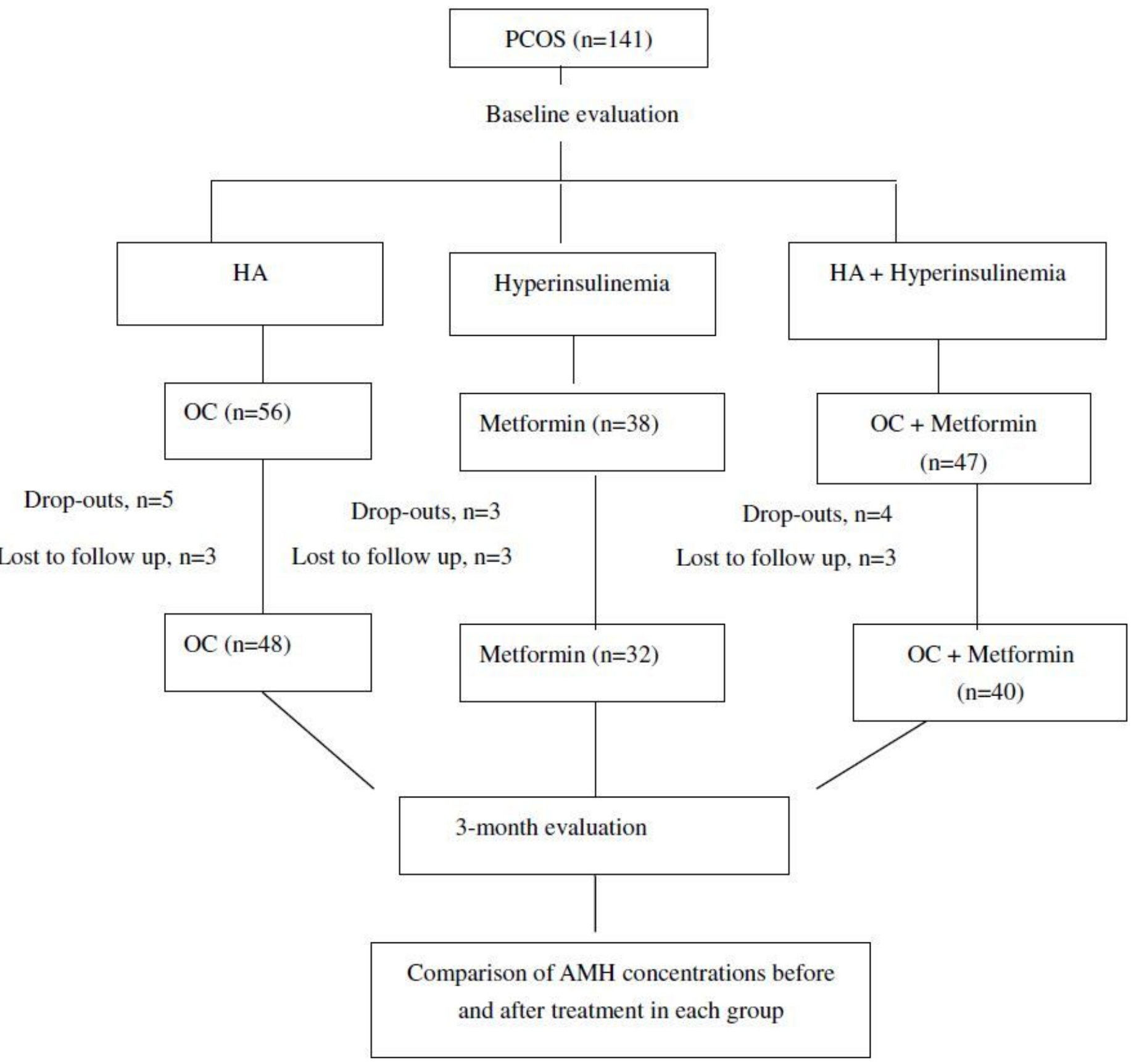

Figure 1

Flow chart of the study. Data are presented as number of participants. OC, oral contraceptives; HA, hyperandrogenism; $\mathrm{AMH}$, anti-Müllerian hormone. 


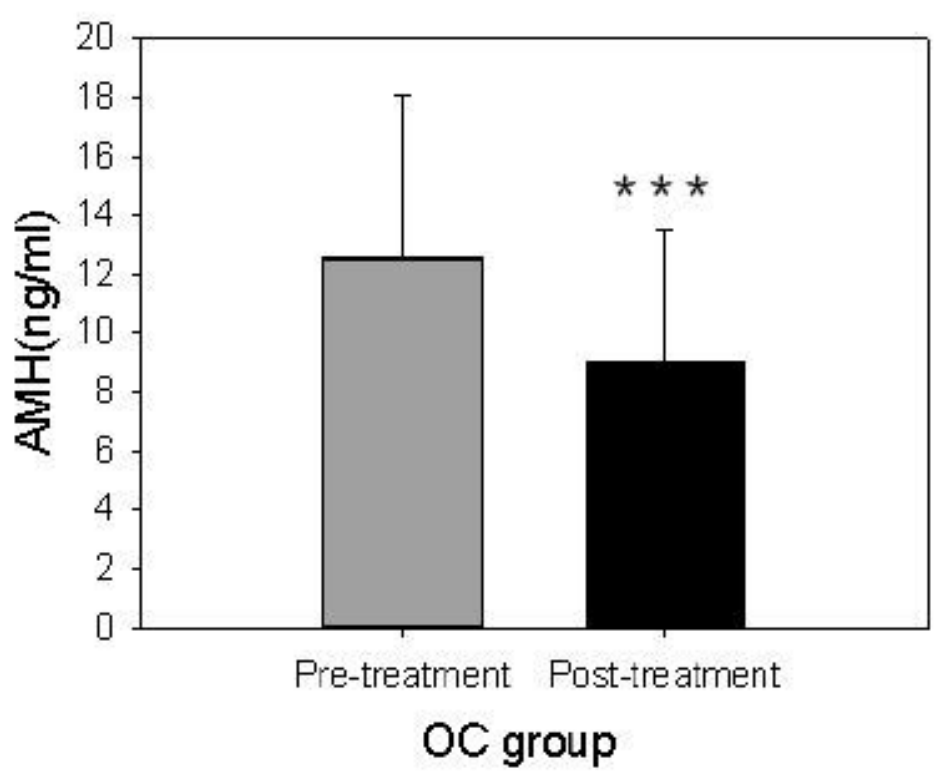

(a)

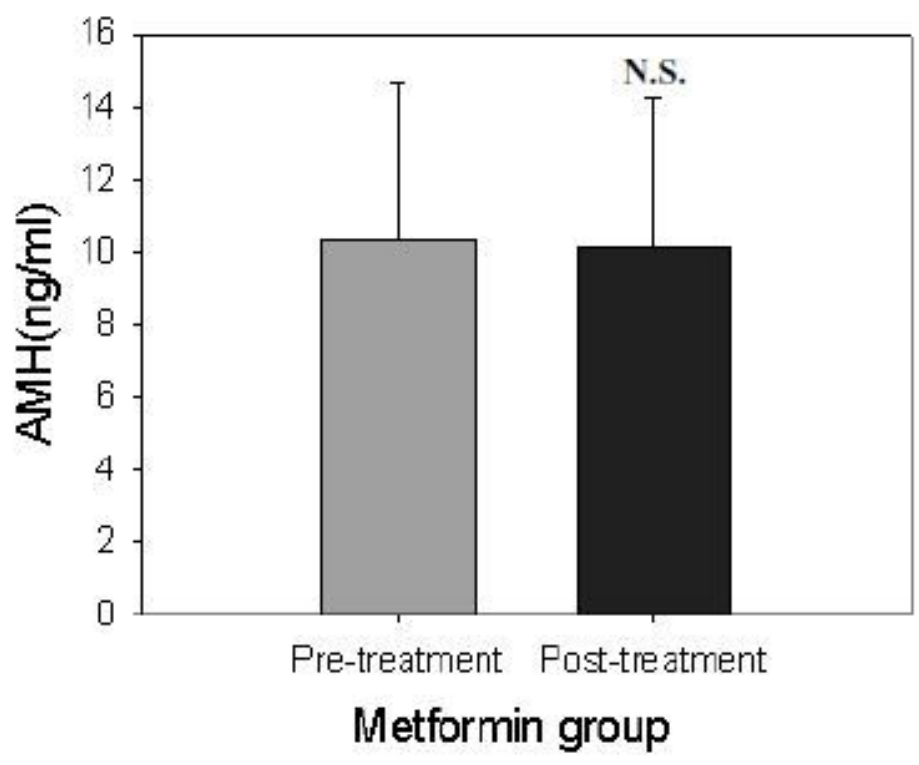

(b)

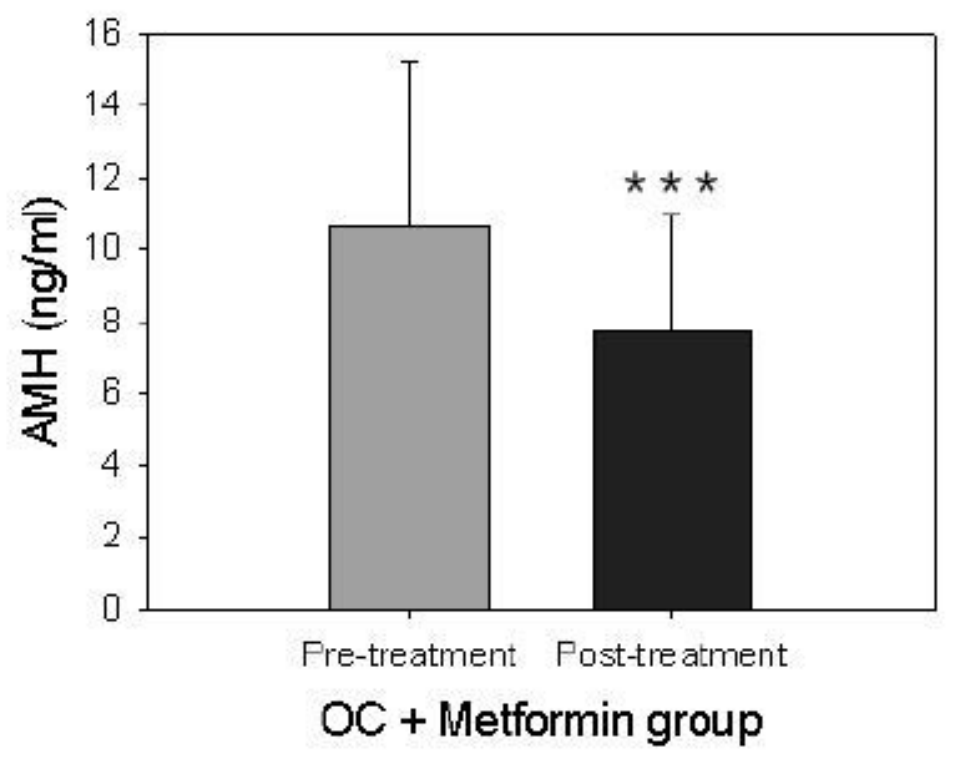

(C)

Figure 2

Comparison of serum AMH concentrations before and after 3 months of treatment within each group. (a) OC group. (b) Metformin group. (C) OC + metformin group. Values are shown as mean \pm SD. OC, oral contraceptives; $\mathrm{AMH}$, anti-Müllerian hormone. 